\title{
FBG Stress-Sensibilized Monitor for Railway Switch Pole On-Line Monitoring
}

\author{
Weilai $\mathrm{LI}^{1 *}$, Jie $\mathrm{LIU}^{2}$, Jianjun $\mathrm{PAN}^{2}$, Jin PANG${ }^{2}$, and Xiaoshan $\mathrm{LU}^{2}$ \\ ${ }^{1}$ National Engineering Lab of Fiber Optic Sensing Technology, Wuhan University of Technology, Wuhan, 430070, China \\ ${ }^{2}$ Key Lab of Fiber Optic Sensing Technology and Information Processing, Ministry of Education, Wuhan University of \\ Technology, Wuhan, 430070, China \\ *Corresponding author: Weilai LI_Ｅ-mail: lwl@whut.edu.cn
}

\begin{abstract}
The fiber Bragg grating (FBG) sensing technology is used to dynamically monitor multiple parameters of railway switch machine poles, including time of movement, direction and quantity of loading and locking force, and states of loading resistance. This paper presents the design and implementation of a railway switch pole strain on-line monitoring system based on the FBG stress-sensibilized monitor for a Siemens S700K switch machine. The ring shape FBG strain gauge and stress-sensibilized methods significantly increased the monitoring sensitivity. Installing approaches adapted the harsh environment in the railway application. The monitoring results showed the high sensitivity and high reliability of this monitoring system. This application provides a long-term and on-line detecting method which could meet railway switch condition monitoring demands of more than 100,000 switch machines in the country.
\end{abstract}

Keywords: Fiber Bragg grating, railway switch, pole, strain, dynamically monitor

Citation: Weilai LI, Jie LIU, Jianjun PAN, Jin PANG, and Xiaoshan LU, "FBG Stress-Sensibilized Monitor for Railway Switch Pole On-Line Monitoring," Photonic Sensors, 2014, 4(3): 269-273.

\section{Introduction}

A railway switch machine is a kind of most important and complicated equipment of the rail structure. Its main function is to control the train direction. Its electric motor changes rotation into translational motion of its switch pole to push or pull the point rails sliding on their paths, that is, the pole executes reciprocating motion. There are two forms of the switch pole, moving point rails to close the left stock rail, that is the pressured stroke, or to close the right stock rail, that is the tensile stroke. When a switch is in the moving stage, its pole is loaded with stress, and when it is in the closing stage, a low stress should be held to keep the position. The quantity of the stress in each stage is supposed to be fitted with its specification. If the stress is overloaded or under-loaded, the point rails would be in the wrong position, which could cause the serious safety problem of the railway. Therefore, reliability monitoring for the stress loaded on the switch pole plays a vital role in ensuring railway transportation safety.

People use the pivot pin with the electric strain gauge to measure pole stress temporarily or measure the electrical parameters of the motor to determine its load. However, as electrical devices, their performance is poor in durability and stability and is poor under the conditions of electrical interference and corrosion [1]. The fiber Bragg grating (FBG) 
sensing technology is of good accuracy, zero drift immunity, anti-electromagnetic interference, and corrosion resistance. It is therefore an ideal technology to monitor pole stress dynamically with long-term reliability.

This paper focuses on a railway switch pole strain on-line monitoring system based on the FBG stress-sensibilized monitor and its application to the switch machine.

The remainder of this article is arranged as follows. Firstly, we describe the operation principle and calibration of an FBG ring strain gauge and an FBG pole stress detector with stress-sensibilized methods for better understanding of the problems and issues associated with this railway switch pole strain monitoring system. Secondly, the structure and installation of this strain monitoring system are presented. Thirdly, some monitoring results are presented to illustrate the accurate monitoring on switch machine.

\section{Operation principle and calibration}

\subsection{FBG ring strain gauge}

The core of this FBG pole stress detector is a ring shape of the FBG strain sensor as shown in Fig. 1. It is a piece of metal gauge with an FBG on it. The FBG is suspended, pre-drawn, and adhered on its non-grating parts. This FBG fixing method is important to avoid the Bragg reflection peak to be chirped [2].

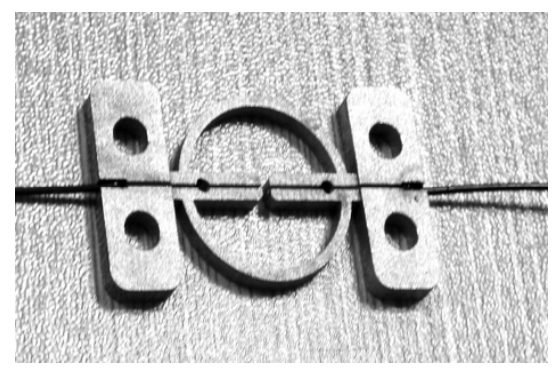

Fig. 1 FBG ring strain gauge.

For mechanical analysis, the structure of this gauge is simplified as a ring as shown in Fig. 2. The elasticity in its detecting direction $\mathrm{AB}$ is designed to be weak, by adjusting the appropriate thickness and width. If putting a small pulling force $F$ on the direction $\mathrm{AB}$, the ring is stretched, and a positive wavelength shift is produced.

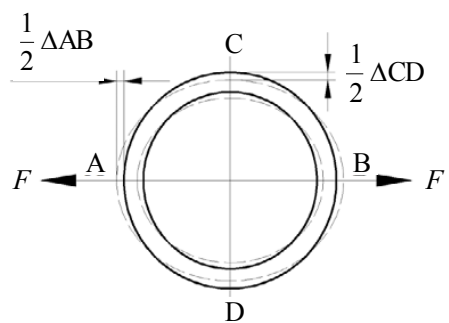

Fig. 2 Mechanical analysis of the deforming ring.

After a loading test, Fig. 3 shows a plot of the wavelength shift versus the load with the pulling force step being $2.5 \mathrm{~N}$, from $0 \mathrm{~N}$ to $20 \mathrm{~N}$. As illustrated in this figure, the FBG wavelength shift ratio is as high as $38 \mathrm{pm} / \mathrm{N}$. Although its elasticity on $\mathrm{AB}$ is weak, the rigidity is good enough to hold a fiber grating.

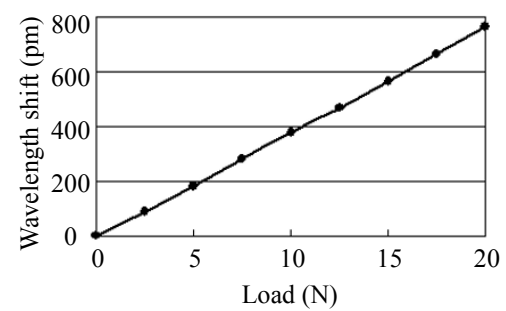

Fig. 3 Elasticity test of a gauge.

Figure 4 shows a strain-wavelength curve of this FBG ring strain gauge by calibration. Its measuring range is $-600 \mu \varepsilon-600 \mu \varepsilon$, and the measuring precision is $\pm 3 \mu \varepsilon$. In the application, the monitoring data are processed as loading force Newton.

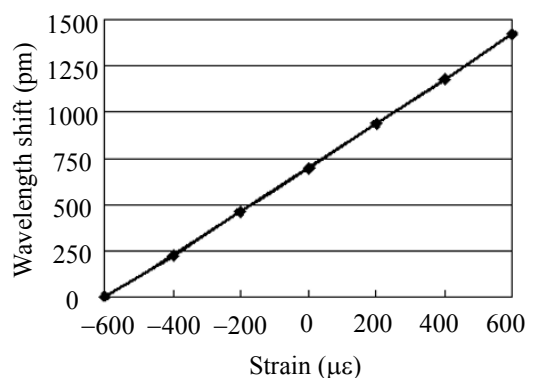

Fig. 4 Wavelength-strain calibration curve of the FBG ring gauge.

\subsection{FBG pole stress detector with the stress- sensibilized method}

With the development of railway electrification 
and high-speed, the S700k type switch machine has been widely used in the railway system. To ensure safety, an FBG pole stress monitoring device with the stress-sensibilized method is applied to a Siemens S700K switch machine.

For this switch machine, the diameter of the pole was $38 \mathrm{~mm}$, moving $220 \mathrm{~mm}$ in stroke, and its normal load was $6 \mathrm{kN}$. Putting a $6-\mathrm{kN}$ pulling force on such a thick steel pole, the strain was supposed to be very small. The experiment confirmed this assumption. A simulated steel pole made with the same steel material and same size was fixed on a testing drawframe, when the tensile force was $10 \mathrm{kN}$, the electric strain gauge adhered on pole's surface showed only $47 \mu \varepsilon$, referring to Fig. 8 . For a bare FBG at a band of $1300 \mathrm{~nm}$, when it was taken as a strain sensor directly, according to our experiment, a micro-strain only produced a 1-pm wavelength shift [3].

A stress-sensibilized method was therefore brought forward. Figure 5 explains this idea. Two sets of clamping blocks with the lengthening rigidity arm are tightly clamped the pole. It is supposed that the distance of two clamping bocks is $D$, where is the strain producing place, while $d$ is the FBG gauge length, which actually measures the strain. $D$ is 7 times as long as $d$. By means of the lengthening rigidity arm, the large deformation from distance $D$ is brought to bear on the short FBG gauge and makes FBG to produce a larger shift. The place of an electric strain gauge adhered on the surface of pole can also be shown in Fig. 5.

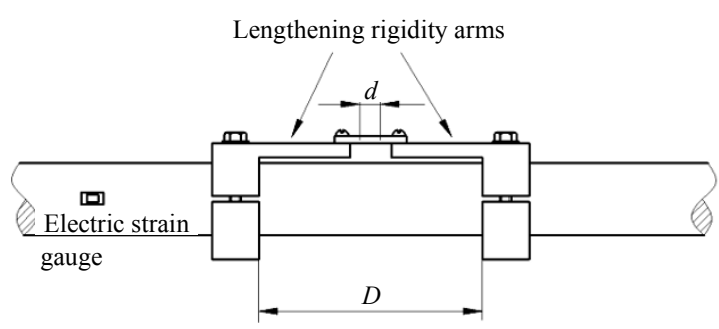

Fig. 5 Stress-sensibilized method.

A measuring device with a simulated pole, an electric strain gauge, and an FBG strain gauge is illustrated in Fig. 6.

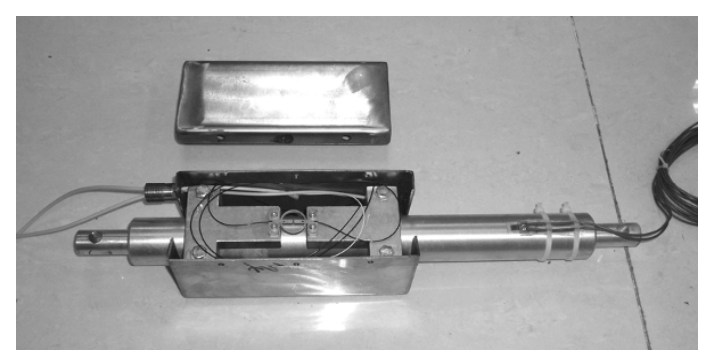

Fig. 6 A monitoring device.

Figure 7 shows the simulated pole explained above doing a loading test on a drawframe. This was the system calibration. The pulling force on the pole was from $0 \mathrm{kN}$ to $12 \mathrm{kN}$, the step was $1 \mathrm{kN}, \mathrm{FBG}$ wavelength shift ratio of the sensibilized gauge was $32 \mathrm{pm} / \mathrm{kN}$.

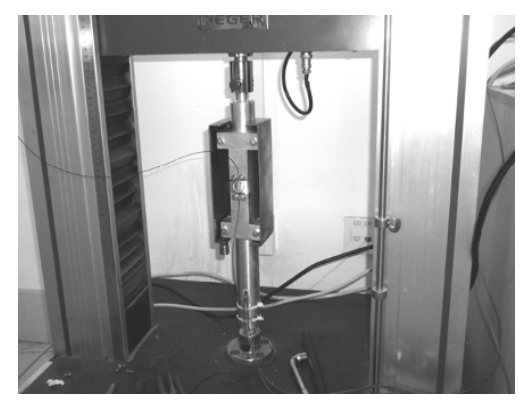

Fig. 7 Loading test on a drawframe.

Figure 8 compares the test results for the strain when the strain is detected by using the electric strain gauge and FBG strain gauge. It can be seen that the strain detected by the FBG is real 7 times higher than the electric one.

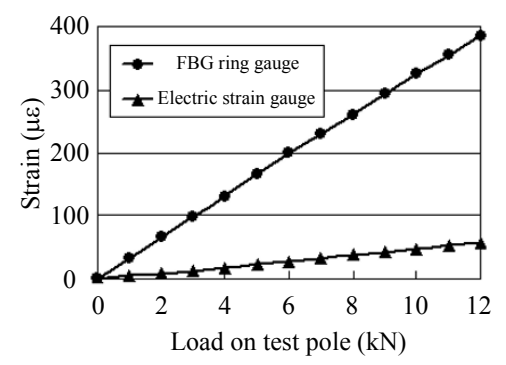

Fig. 8 Calibration results.

\section{Structure and installation}

Figure 9 illustrates a scheme of the railway switch pole strain monitoring system, where 1 represents the pole, 2 is for a shell, 3 represents upper clamping blocks with the lengthening rigidity arm, 4 represents clamping block bolts, 5 represents 
nether clamping blocks, 6 represents an FBG strain gauge, 7 represents screws, 8 represents another FBG strain gauge for temperature compensation with a different Bragg wavelength, 9 represents the transmission fiber, 10 represents fiber connecting boxes, 11 represents the armored fiber cable, 12 represents a demodulator, 13 represents an industrial personal computer (IPC) for data processing, 14 represents clients of switch management, 15 and 16 are the next pole strain monitors.

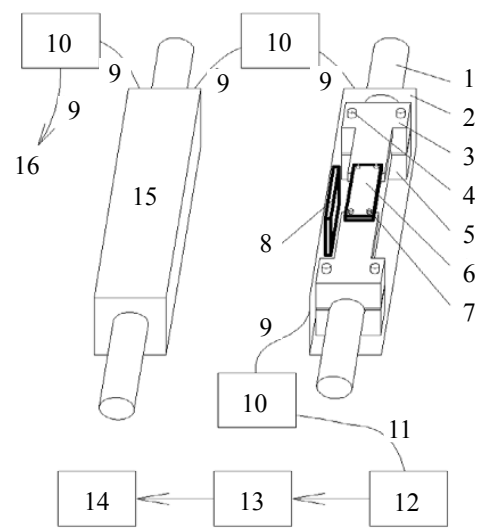

Fig. 9 Scheme of the switch pole strain monitoring system.

In the switch monitoring application, as shown in Fig. 10, the monitoring place on the pole is indicated by an arrow.

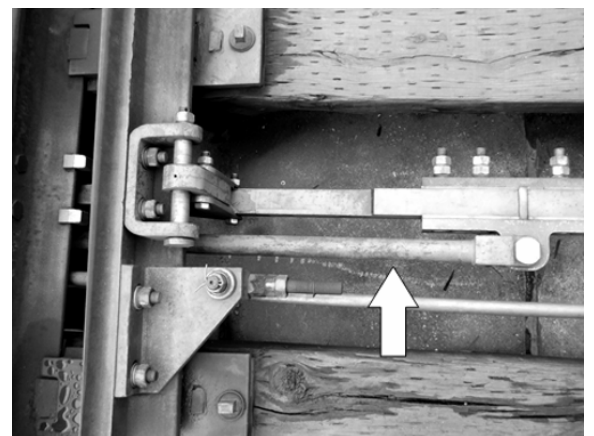

Fig. 10 Place at which a monitoring device is fixed.

Figure 11 shows the installation of the strain monitoring device in the harsh environment with oil and dust and in the open areas. Because the applying place was in an urban railway line, only stops train operation in the night. The FBG unite was protected safely by a stainless steel shell, and electric insulation approaches were accepted for the operation of the track circuit. Since the device was moved $220 \mathrm{~mm}$ with the pole in every stroke, the fiber was holed by a looped rubber paper.

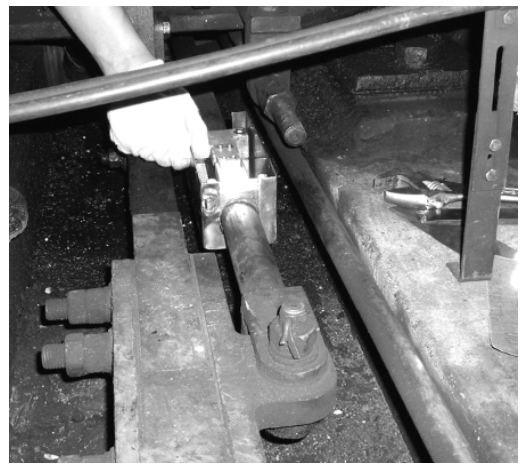

(a)

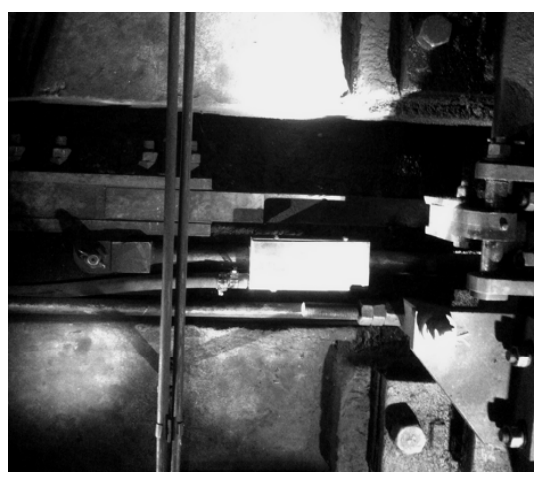

(b)

Fig. 11 Installing an FBG strain monitoring device for (a) installation process and (b) appearance after installation.

\section{Monitoring results}

The monitoring contents are as follows: time of pole movement, direction and quantity of loading force, and the states of loading resistance. They were recorded dynamically by the system. When the switch pole was on the pressured stroke, the operation time was 5 seconds, the maximum load was $-5.9 \mathrm{kN}$, and the average load during this 5 seconds was $-3.3 \mathrm{kN}$. The vibration caused by resistance was obvious. Its period was 0.1 second, and its range was from $0.5 \mathrm{kN}$ to $1.6 \mathrm{kN}$. In the closing stage, a locking force was kept about $-0.6 \mathrm{kN}$. Figure 12 shows the curve.

On the other hand, during the tensile stroke, referring to Fig. 13, the operation time was also 5 seconds, and the maximum load was $6.8 \mathrm{kN}$, larger than that of the pressured stroke. The average load was $5.5 \mathrm{kN}$. The vibration period was 0.1 second, 
and its range was from $0.7 \mathrm{kN}$ to $2 \mathrm{kN}$. The locking force was about $0.2 \mathrm{kN}$.

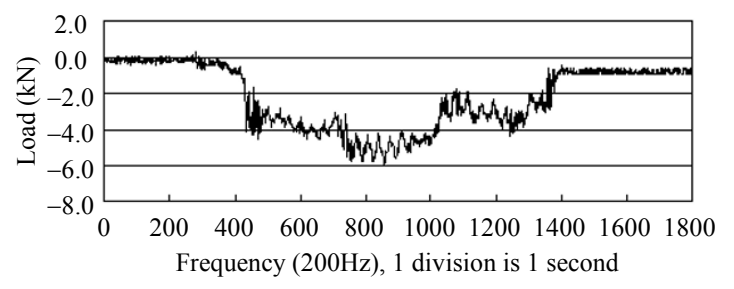

(a)

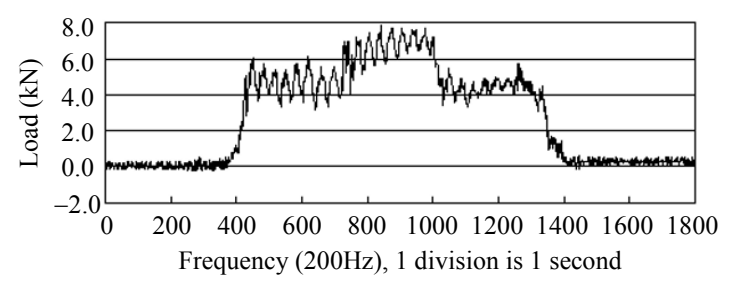

(b)

Fig. 12 Monitoring results of the (a) pole pressured stroke and (b) pole tensile stroke.

Figure 13 shows a vibrative curve taken from a train passed by the monitoring device. It confirms the high sensitivity of this monitoring system.

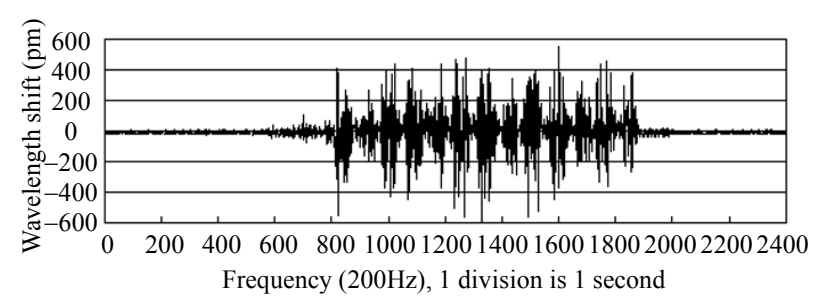

Fig. 13 Vibrative curve when a train is passing by.

\section{Conclusions}

This application provides a dynamical and sensitive increasing method to do multiple and accurate monitoring on the switch machine. It could therefore meet railway switch pole stress monitoring demands of more than 100,000 switch machines in the country. As it is known, lots of the novel optical fiber sensor (OFS) theory and method are being put forward. However, their application lags behind these lab works. This application tries to explain that as long as people focus on engineering works, OFS's engineering application could be useful and popular.

\section{Acknowledgment}

The authors gratefully acknowledge the financial support of this work by the National Science Foundation of China, Numbered 61290311.

Open Access This article is distributed under the terms of the Creative Commons Attribution License which permits any use, distribution, and reproduction in any medium, provided the original author(s) and source are credited.

\section{References}

[1] D. Jiang and W. He, "Review of application for FBG sensors," Optoelectronics \& Lasers, 13(4): 420-426, 2002.

[2] W. Li, Y. Zhang, Q. Wang, J. Pan, J. Liu, and C. Zhou, "Displacement monitor with FBG deforming ring and its application in high speed railway," OFS2012 22nd International Conference on Optical Fiber Sensors, Beijing, China, October 14, vol. 8421, pp. 8421AU-1-8421AU-4.

[3] W. Li, X. Huang, J. Cheng, and J. Pan. "Strain-temperature monitor of high speed railway switch by fiber Bragg grating gauges," The Fifth International Symposium on Advanced Optical Manufacturing and Testing Technologies, Dalian, China, October 13, 2010, vol. 7656, pp. 76565E. 